\title{
QR CODE WATER MARKING ALGORITHM BASED ON DWT AND COUNTERLET TRANSFORM FOR AUTHENTICATION
}

\author{
A.S. Tonne ${ }^{1}$ and V.S.Bale ${ }^{2}$
}

\begin{abstract}
Digital Watermarking is a technology used for the copyright protection of digital applications. Watermarking is a concept of embedding a special symbol, watermark, into an electronic document so that a given piece of copyright information is permanently tied to the data. It is an effective way to protect copyright of multimedia data even after its transmission. In recent years image watermarking techniques are grown-up. In this science era with the development of technology and internet, the protection and authentication of data is essential. Watermarking techniques provide solution to this problem. QR code being so versatile because of its structural flexibility that it leads to so many diverse field for research such as increasing data capacity, security applications such as different kinds of watermarking. We have used QR code as cover image and as secret image to protect it from other users. This paper is representing a new watermarking technique with $\mathrm{QR}$ code to protect the secret image by using DWT and Counterlet Transform. In the method described here the image is first encrypted in random matrix, then it is invisibly watermarked in cover image and no information about the secret image and cover image is needed for extraction of secret image, so it more secure. Counterlet Transform is advance technique and its performance is higher than DWT for water mark embedding and extraction.
\end{abstract}

KEYWORDS: Watermarking, DWT, QR code, Watermark embedding, Watermark extraction, Counterlet Transform

\section{INTRODUCTION}

Increase in use of Digital Media, raises the problem of data protection and authentication. Data can be can be easily copied. Digital Watermarking Technique gives the best solution to protect data. In this technique the data is hidden within the cover image so stranger cannot get it without having proper guidance. Many methods used for the invisible Digital Watermarking required some information about the cover image. But we do not require it for secret image extraction, so it is more secure. We are using $\mathrm{QR}$ code which itself hide the information but which can be easily scanned .We can use this method to hide image in QR code and to hide QR code in an image. In both the cases the information is detectable under the influence of various attacks.

${ }^{1}$ Department of ECE, M.S. Bidve Engineering College, Latur, INDIA

${ }^{2}$ Department of ECE, M.S. Bidve Engineering College, Latur, INDIA 


\subsection{Discrete Wavelet Transform}

It decomposes the image into different frequency ranges such as low frequency, middle frequency and high frequency. In same way the image can further be decomposed into $n$ levels. Here we are using 3level decomposition for better result.

In two dimensional applications, for each level of decomposition, we first perform the DWT in the vertical direction, followed by the DWT in the horizontal direction. After the first level of decomposition, there are 4 sub-bands: LL, $\mathrm{LH}, \mathrm{HL}$, and HH. For each successive level of decomposition, the LL sub-band of the previous level is used as the input. To perform second level decomposition, the DWT is applied to LL and so on.

\begin{tabular}{|c|c|c|}
\hline LL2 & HL2 & \multirow{2}{*}{ HL } \\
\hline \hline LH2 & HH2 & \\
\hline \multicolumn{2}{|c|}{ LH } & HH \\
& & \\
& & \\
\hline
\end{tabular}

Figure 1: DWT decomposition

\subsection{Quick Response Code}

It is two dimensional barcode consists of black modules arranged in white background. It can store large alphanumeric information and easily readable by scanner. A QR code is capable of being read in 360 degree from any direction thus eliminating interference. The QR Code system has become admired outside the automotive industry due to its fast readability and greater storage capacity than that of the UPC barcode.

- It is higher information density.

- $\quad$ It is small in size than that of Barcode.

- $\quad$ Data can be restored even if the symbol is partially dirty or damaged.

Some features that describe how QR code is better in comparison with conventional barcodes have been listed out in following table

Table 1: Comparison of QR code and Barcode

\begin{tabular}{|c|c|}
\hline QR Code & Barcode \\
\hline 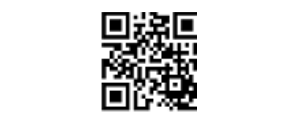 & \\
\hline $\begin{array}{l}\text { UPTO } 7089 \text { numeric } \\
\text { digits }\end{array}$ & $10-20$ digits \\
\hline $\begin{array}{l}40 \text { digit Numeric (approx } \\
5 \mathrm{~mm} 5 \mathrm{~mm} \text { ) }\end{array}$ & $\begin{array}{l}10 \text { digit Numeric (approx } \\
50 \mathrm{~mm} 20 \mathrm{~mm} \text { ) }\end{array}$ \\
\hline $\begin{array}{l}\text { Supports } 360 \mathrm{~d} \\
\text { reading }\end{array}$ & Horizontal reading \\
\hline
\end{tabular}


QR code is used for Advertising, Business cards, Social networking, Branding, registration.

\section{PROPOSED METHOD}

\subsection{Watermark Embedding}

The step of embedding process are outlined as follows

\section{Step of watermark image with secret key}

(1) The watermark image was produced as a bit sequence of watermark S. The data and background values were set to 1 and -1 , respectively.

$\mathbf{S}=\left\{s_{i}, 1 \leq i \leq N\right\}, s_{i} \in\{-1,1\}$

Where, $\mathrm{N}$ is the total number of pixels in the watermark image

(2) The pseudo-random sequence $(\mathrm{P})$ whose each number can take a value either 1 or -1 was randomly generated with a secret key for embedding and extracting of the watermark.

$\mathrm{P}=\left\{p_{i}, 1 \leq i \leq N\right\}, p_{i} \in\{-1,1\}$

\section{Step of $Q R$ code image}

(1) The two-level DWT of $M \times M$ image $\left(t_{i}\right)$ i t was computed for QR code image.

(2) A watermark was then embedded in sub band $\mathrm{LH}_{2}$ or $\mathrm{HL}_{2}$ or $\mathrm{HH}_{2}$. According to the rule:

$t_{i}^{i}=t_{i}+\alpha, p_{i}, s_{i}, i=1,2, \ldots, N$

Where, $t_{\mathrm{i}}$ is input image, ' $t_{i}^{f}$ is output image with watermark. $\alpha$ is a magnitude factor which is a constant determining the watermark strength.

(3) After that, the inverse DWT (IDWT) was then applied to obtain the watermarked image.

(4) Compute PSNR

The quality of the watermarked video compared to

original video was measured based on the Peak SignaltoNoiseRatio(PSNR). 


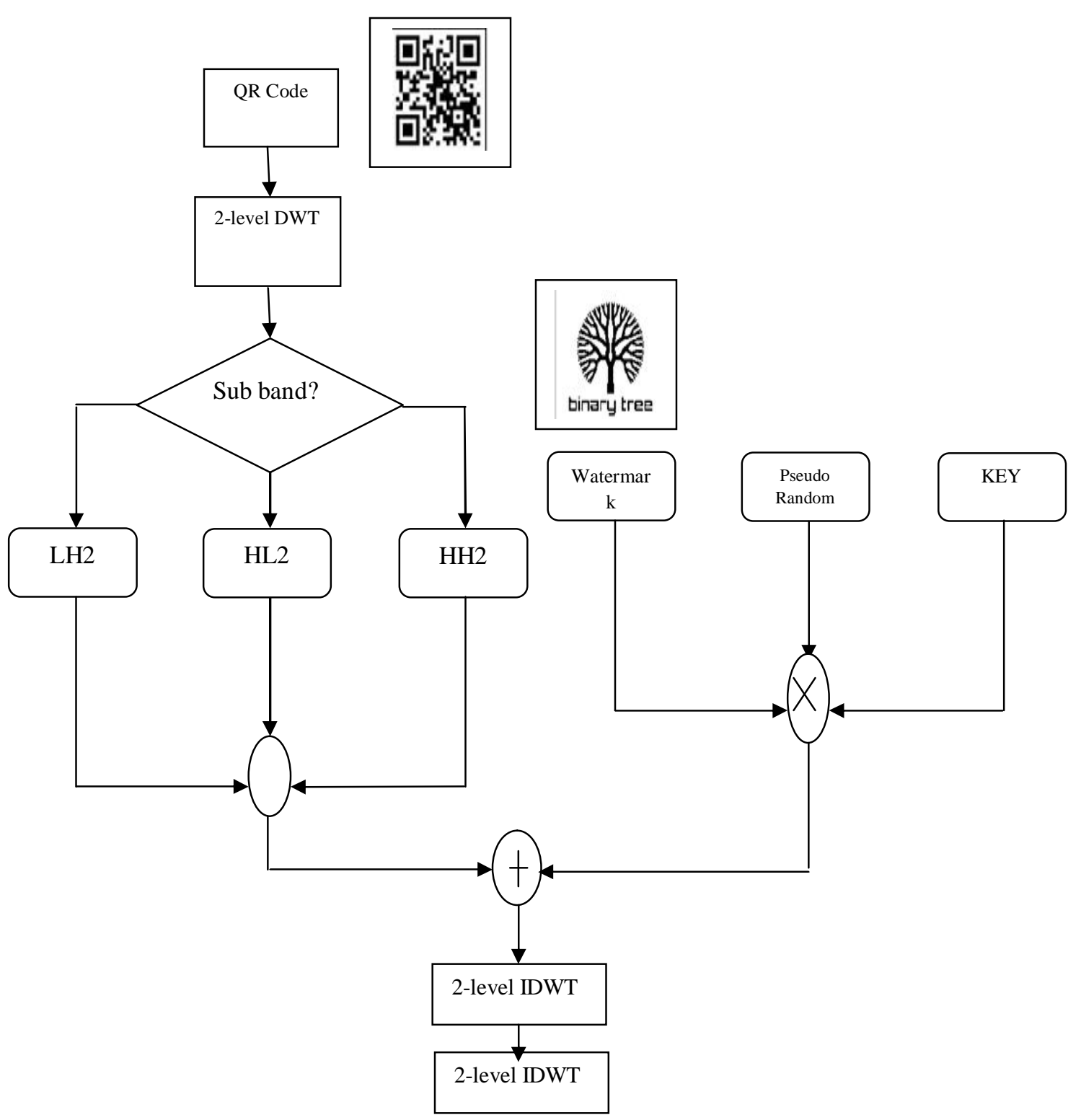

Figure 2: Watermark Embedding Process

$$
P S N R=10 \log \frac{\left(2^{b}-1\right)}{\frac{1}{m \times n} \sum_{i=0}^{m-1} \sum_{j=0}^{n-1}(O(i, j)-R(i, j))^{2}}
$$

\subsection{Watermark Extraction}

The watermark extraction algorithm did not use the original $Q R$ code image. A prediction of the original value of the pixels is however needed. Thus, a prediction of the original value of the pixels was performed using noise 
elimination technique. In this paper, we use an averaging $3 \times 3$ mask whose elements were fixed to $1 / 9$. The extraction process are outlined as follows

(1) The predicted image $\hat{t}_{i}$ could be obtained by smoothing the input image $t_{i}^{*}$ with a spatial convolution mask. The prediction of the original value can be defined as:

$f_{i}=\frac{1}{\operatorname{cxc}} \sum_{i}^{\operatorname{cxc}} t_{i}^{*}$

Where, $\mathrm{c}$ is the size of the convolution mask. The watermarked image and the predicted image were DWT transformed independently

(2) The estimate of the watermark $S_{1}$ is indicated by the difference between $t_{i}^{*}$ and $\hat{t}_{\mathrm{i}}$ as:

$\delta=t_{i}^{\prime \prime}-t_{1}=\alpha, p_{i}, s_{1}$

(3) The sign of the difference between the predicted and the actual value is the value of the embedded bit:

$\operatorname{sgn}\left(\delta_{i}\right)=p_{i}, \hat{s}_{l}$

(4) COMPUTE NC

To evaluate the performance of the algorithm, a similar measurement between the original watermark $(S)$ and the extract watermark $\left(S^{\prime}\right)$ was computed by using normalized correlation (NC).

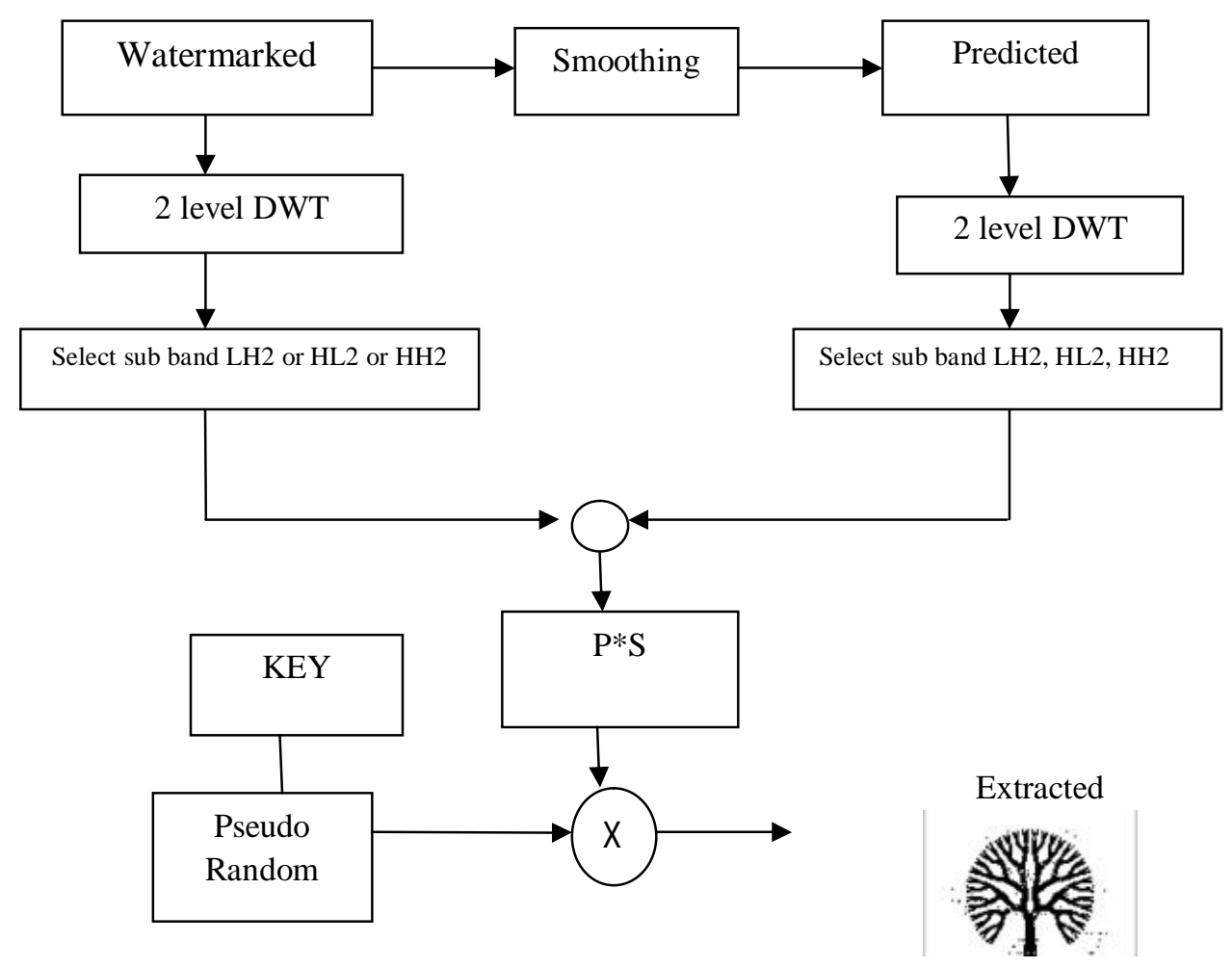

Figure 3: Watermark Extracting Process using DWT 
NC and PSNR having mathematical representation as,

$$
N C=\frac{\sum_{i=1}^{M} S_{i} S_{i}^{\prime}}{\sum_{i=1}^{M} S_{i}^{2}}
$$

\section{CONTOURLET TRANSFORM:}

Contourlets form a multiresolution directional tight frame designed to efficiently approximate images made of smooth regions separated by smooth boundaries. The Contourlet transform has a fast implementation based on a Laplacian Pyramid decomposition followed by directional filter banks applied on each bandpass sub band. Contourlet transform is a double filter bank structure. It is implemented by the pyramidal directional filter bank (PDFB) which decomposes images into directional subbands at multiple scales. In terms of structure the contourlet transform is a cascade of a Laplacian Pyramid and a directional filter bank.

Block diagram of embedding and extraction of data in contourlet domain is shown in Fig.4.The secret data is first encrypted using proper algorithm and then embedding algorithm is applied.

\subsection{CONTOURLET}

Contourlet form a multiresolution directional tight frame designed to efficiently approximate images made of smooth regions separated by smooth boundaries. The Contourlet transform has a fast implementation based on a Laplacian Pyramid decomposition followed by directional filterbanks applied on each bandpass subband.

Table 2: PSNR vs. NC

\begin{tabular}{|c|c|c|c|}
\hline $\begin{array}{l}\begin{array}{l}\text { Sub } \\
\text { band }\end{array} \\
\end{array}$ & PSNR & NC & $\begin{array}{l}\text { Extracted } \\
\text { watermarl }\end{array}$ \\
\hline LH & 43.0615 & $\begin{array}{l}0.95 \\
25\end{array}$ & $\begin{array}{l}\text { vyly } \\
\text { 今y } \\
\text { binary tree }\end{array}$ \\
\hline HL & 43.1514 & $\begin{array}{l}0.96 \\
11\end{array}$ & $\begin{array}{l}\text { vyle } \\
\text { जy } \\
\text { binary tree }\end{array}$ \\
\hline $\mathrm{HH}$ & 44.2675 & $\begin{array}{l}0.99 \\
16\end{array}$ & 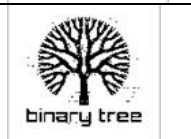 \\
\hline
\end{tabular}


Table3: Watermark Extraction

\begin{tabular}{|c|c|c|}
\hline 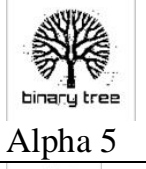 & 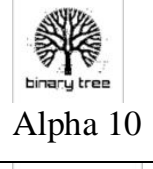 & $\begin{array}{l}\text { syie } \\
\text { vity } \\
\text { tinary tree } \\
\text { Alpha } 15\end{array}$ \\
\hline 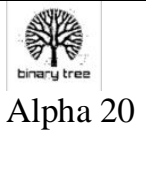 & $\begin{array}{l}\text { syes } \\
\text { tonary tree } \\
\text { Alpha } 25\end{array}$ & $\begin{array}{l}\text { Syle } \\
\text { binary tre } \\
\text { Alpha } 30\end{array}$ \\
\hline $\begin{array}{c}\text { Ayve } \\
\text { tof } \\
\text { tinary tree } \\
\text { Alpha } 35\end{array}$ & 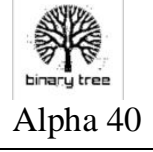 & 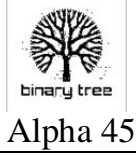 \\
\hline
\end{tabular}

Text Image

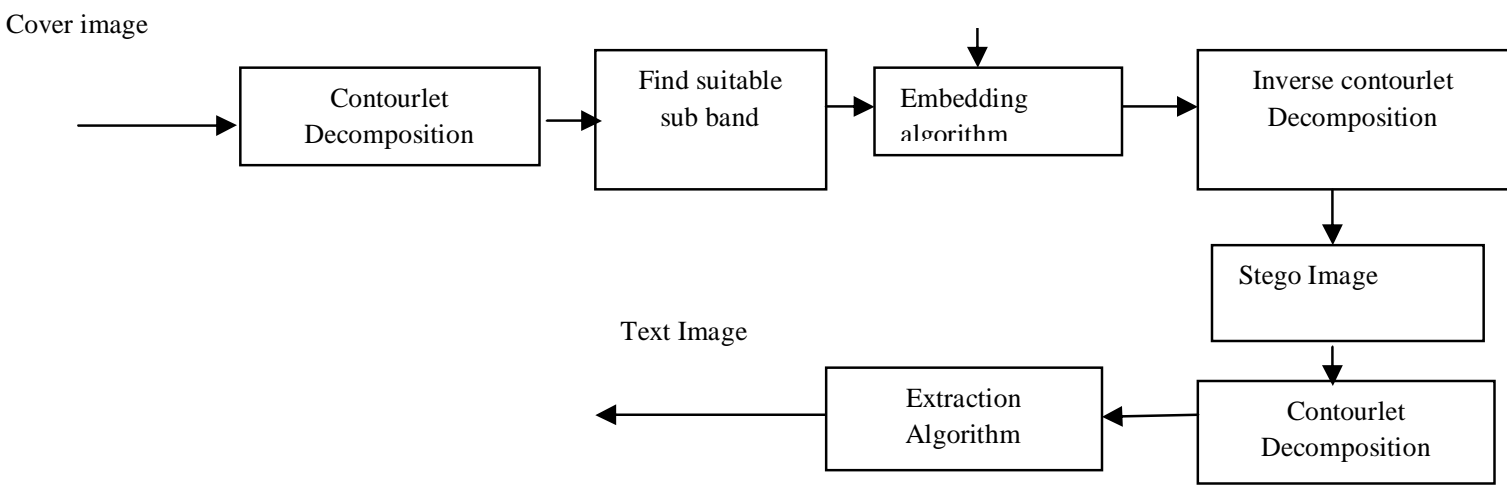

Figure 4: Watermark Embedding \& Extracting Process using COUNTOURLET TRANSFORM

\section{SIMULATION RESULTS}

Table 3: PSNR and NC of QR decode Image using DWT

\begin{tabular}{|l|l|l|c|}
\hline $\boldsymbol{\alpha}$ & PSNR & NC & $\begin{array}{l}\text { DECODE } \\
\text { Code }\end{array}$ \\
\hline 5 & 46.1926 & 0.9794 & $\checkmark$ \\
\hline 10 & 40.1720 & 0.9903 & $\checkmark$ \\
\hline 15 & 36.6502 & 0.9942 & $\checkmark$ \\
\hline 20 & 34.1514 & 0.9963 & $\checkmark$ \\
\hline 25 & 32.3132 & 0.9972 & $\checkmark$ \\
\hline 30 & 30.6296 & 0.9981 & $\checkmark$ \\
\hline 35 & 29.2906 & 0.9991 & $\checkmark$ \\
\hline 40 & 28.1308 & 0.9993 & $\checkmark$ \\
\hline 45 & 27.1078 & 0.9994 & $\checkmark$ \\
\hline
\end{tabular}


Table 4: PSNR and NC of QR decode Image using Contourlet Transform

\begin{tabular}{|l|l|l|c|}
\hline $\boldsymbol{\alpha}$ & PSNR & NC & $\begin{array}{l}\text { DECODE } \\
\text { Code }\end{array}$ \\
\hline 5 & 46.4564 & 0.9810 & $\mathbf{Q R}$ \\
\hline 10 & 40.4359 & 0.9936 & $\checkmark$ \\
\hline 15 & 36.9141 & 0.9969 & $\checkmark$ \\
\hline 20 & 34.4153 & 0.9985 & $\checkmark$ \\
\hline 25 & 32.4771 & 0.9991 & $\checkmark$ \\
\hline 30 & 30.8935 & 0.9993 & $\checkmark$ \\
\hline 35 & 29.5545 & 0.9994 & $\checkmark$ \\
\hline 40 & 28.3946 & 0.9996 & $\checkmark$ \\
\hline 45 & 27.3717 & 0.9996 & $\checkmark$ \\
\hline
\end{tabular}

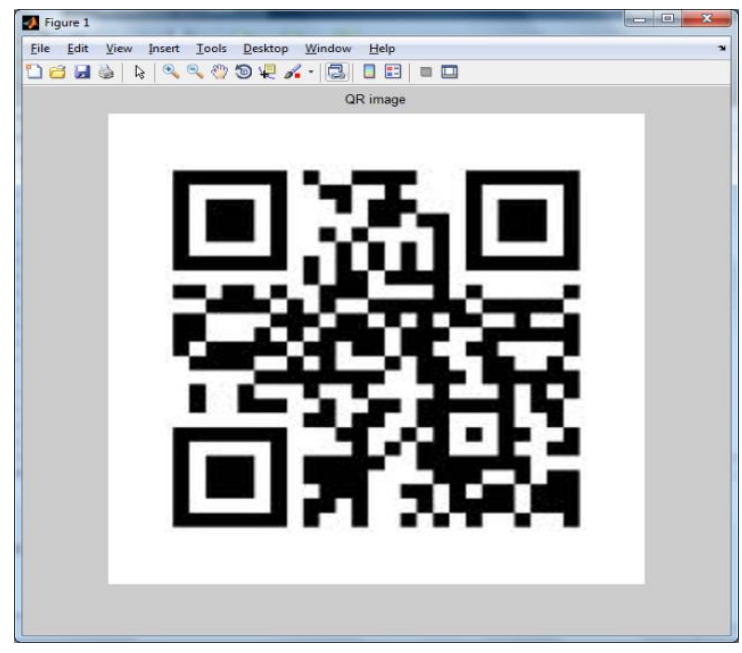

Figure 5: QR image

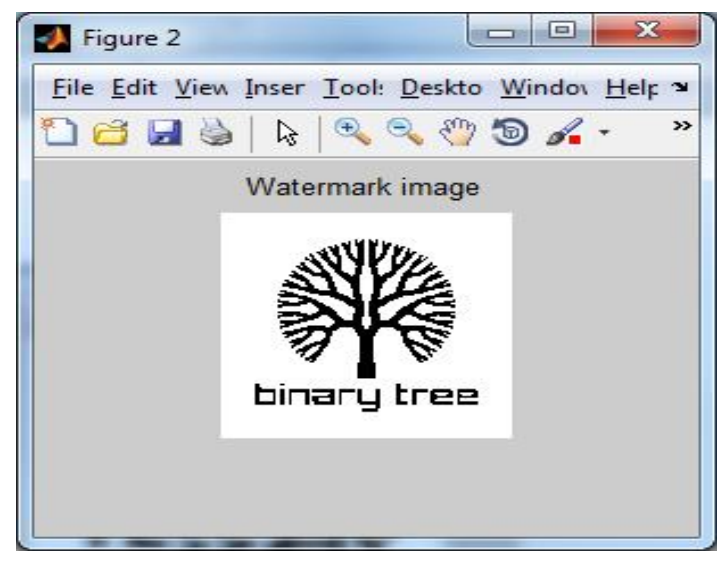

Figure 6: Watermarked image 


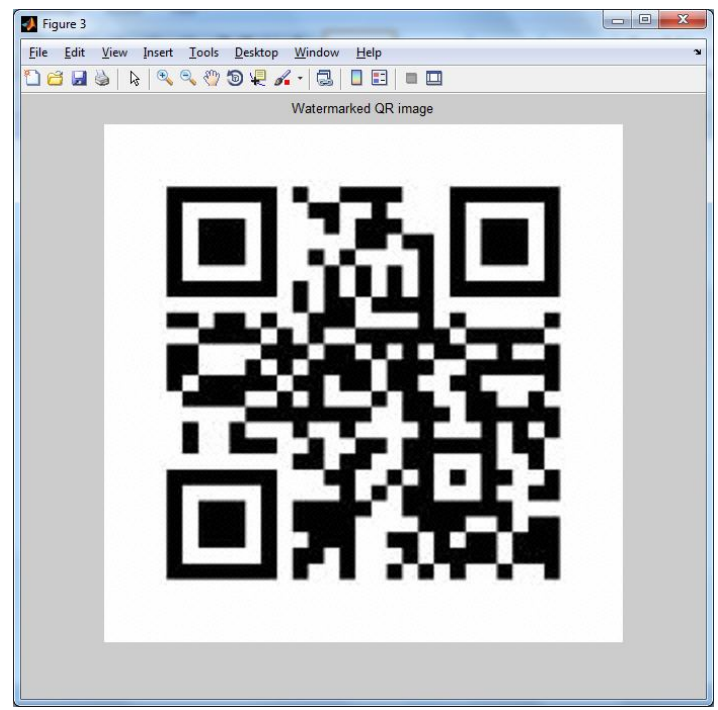

Figure 7: Watermarked QR image

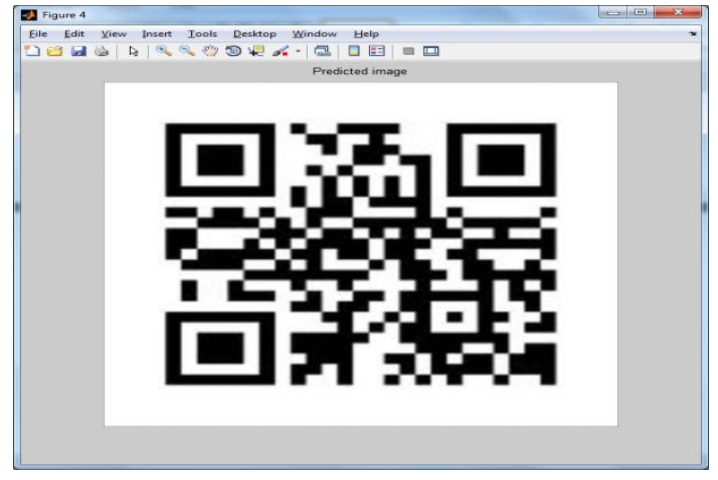

Figure 8: Predicted image

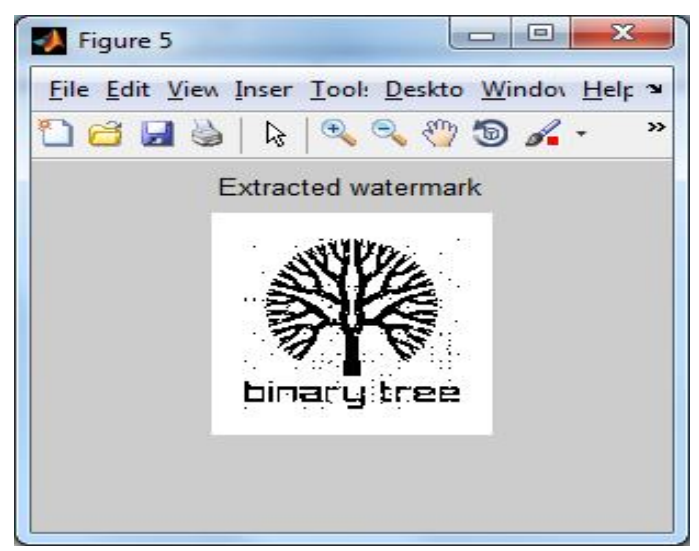

Figure 9: Extracted watermark 


\section{EXTENSION RESULTS}

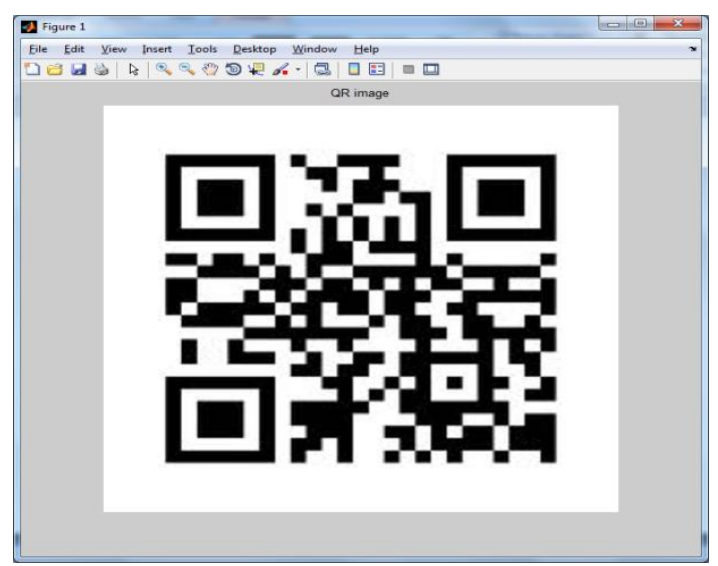

Figure 10: QR image (Contourlet transform)

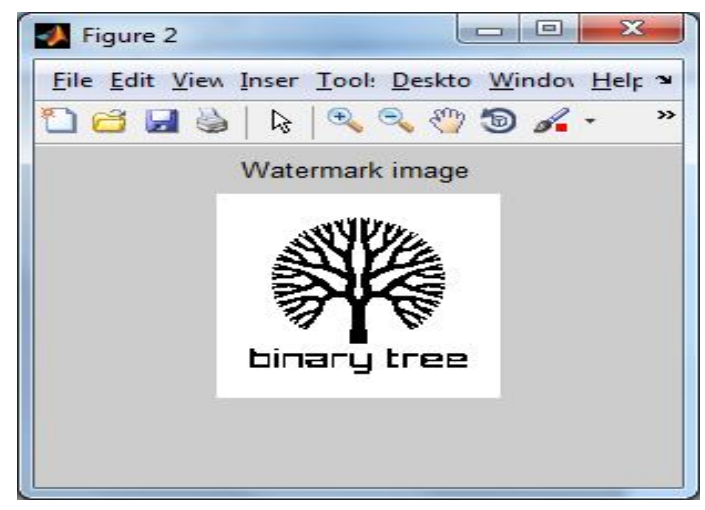

Figure 11: Watermarked image (Contourlet transform)

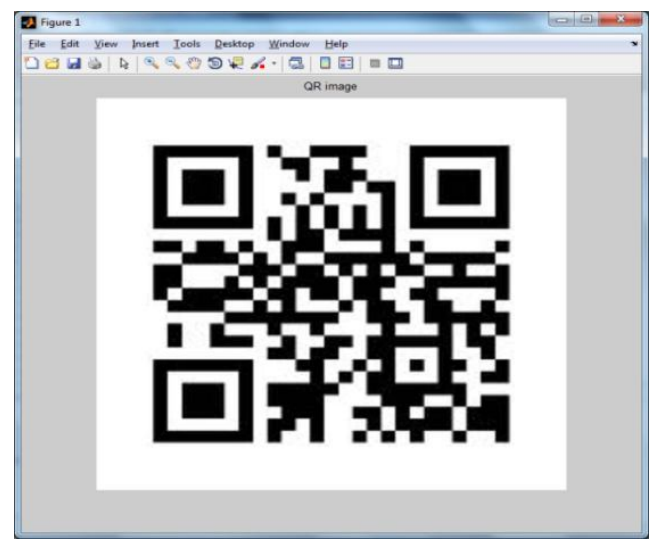

Figure 12: Watermarked QR image (Contourlet transform) 


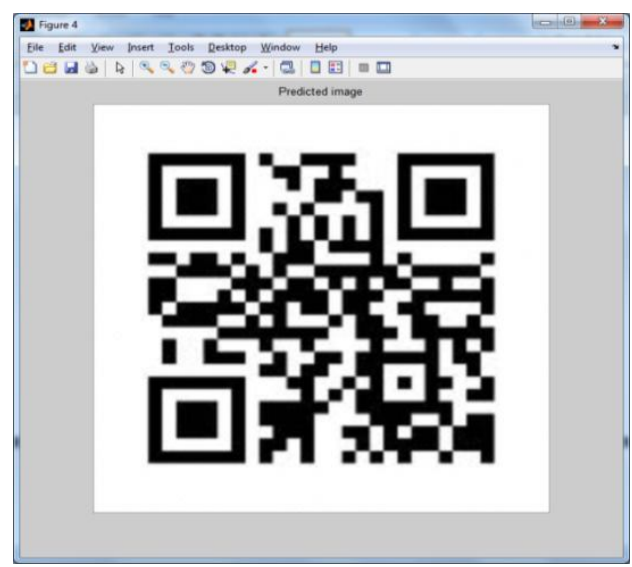

Figure 13: Predicted image (Contourlet transform)

\section{CONCLUSION}

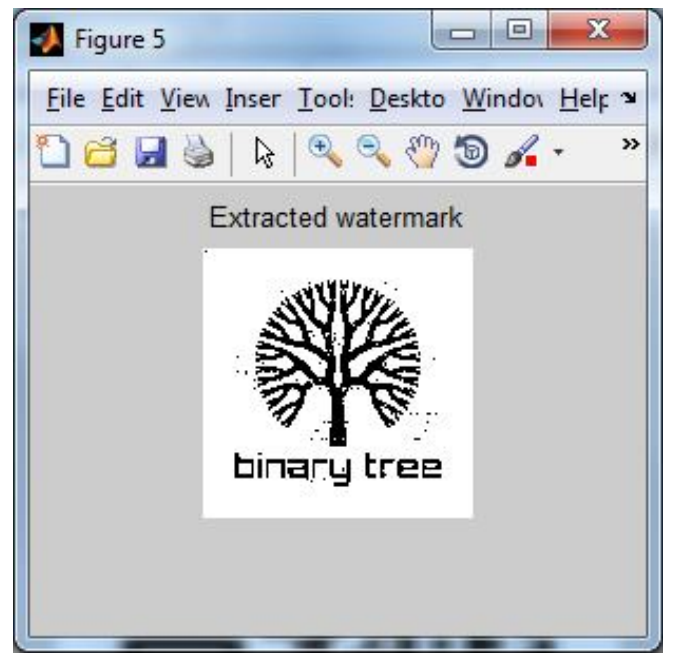

Figure 14: Extracted image (Contourlet transform)

This paper presented a digital watermarking technique which improves the characteristics of watermarking which is based on DWT \& Counterlet Transform. Whereby a binary image is watermarked an embedded in a QR Code image. The embedding process is presented in a $\mathrm{LH}, \mathrm{HL}$ or $\mathrm{HH}$ sub band based on wavelet transform. The experimental results demonstrated that the algorithm can be recovering the watermark with an acceptable visual quality. The objective measures such as PSNR, NC and MAE are subject to magnitude factor. As the future work, the quality of the watermarked video Compared to original video was measured based on the Peak Signal to Noise Ratio (PSNR), Normalized Correlation (NC) and Mean Absolute Error (MAE). Performance of Counterlet Transform as compared to DWT is better in noise reduction, edge preservation and image enhancement.

\section{REFERENCES}

[1] "Denso wave incorporated, "http://www.densowave.com/qrcode/indexe. html. 
QR Code Water marking Algorithm Based on Dwt and Counterlet Transform for Authentication 406

[2] Lumini A and Maio D (2000) A wavelet-based image watermarking scheme In:Proc. Int.Conf. Information Technology: Coding and Computing. pp.122-127.

[3] Alattar AM(2004) Reversible watermark using the difference expansion of a generalized integer transform. IEEE Trans. Image Process. 13: 1147- 1156.

[4] Jun-Chou Chuang, Yu-Chen Hu \& Hsien-Ju Ko. A Novel Secret Sharing Technique Using QR Code, International Journal of Image Processing (IJIP), Volume (4) : Issue (5), pp. 468-475, 2010.

[5] "QRCode," http://en.wikipedia.org/wiki/QR code.

[6] ENSO Wave Incorporated. What is a QR Code?, 2013. http://www.qrcode. com/en/. Accessed 10 Feb 2013.

[7] M. J. Shensa, "The Discrete Wavelet Transform Wedding À Trouse and Mallat Algorithms," IEEE Trans. on Signal Processing, vol. 40, no. 10, October 1992 P. Kieseberg, code security. In Proceedings of the 8th

International Conference on Advances in Mobile 